\title{
Uma Abordagem da Suplementação de Vitamina D e Cálcio em Pacientes Submetidos à Cirurgia Bariátrica
}

\section{An Approach of Vitamin D and Calcium Supplementation in Patients Submitted to Bariatric Surgery}

\author{
Dora de Castro Agulhon Segura ${ }^{1}$ \\ Paula Emanuelly Schneider ${ }^{2}$ \\ Djeice Diane Heck ${ }^{2}$ \\ Bruna Marcusso Rodrigues ${ }^{2}$ \\ Danile Maria Gomes ${ }^{2}$
}

\section{RESUMO}

Objetivo: Avaliar o efeito de uma suplementação de cálcio e vitamina $\mathrm{D}$ em indivíduos submetidos à cirurgia bariátrica. Metodologia: Tratou-se de um estudo descritivo, sendo entrevistados 52 indivíduos, de ambos os gêneros, sem fator idade, submetidos à gastroplastia redutora tipo $\mathrm{Y}$ de Roux, com tempo mínimo de pós-operatório de 12 meses. Foi aplicado um questionário voltado à investigação da suplementação de cálcio e vitamina D. Resultados: Constatou-se que $28,84 \%$ dos indivíduos responderam que suplementavam cálcio e $57,69 \%$ suplementavam vitamina D. Na comparação entre os gêneros, $7,69 \%$ dos homens e $35,89 \%$ das mulheres afirmaram suplementar cálcio, $30,76 \%$ dos homens e $66,66 \%$ das mulheres responderam suplementar vitamina $\mathrm{D}$. O resultado bioquímico da última dosagem de vitamina $\mathrm{D}$ demonstrou homens com $43,3 \mathrm{ng} / \mathrm{mL}$ e mulheres com $32,6 \mathrm{ng} / \mathrm{mL}$, a averiguação do cálcio apresentou $9,1 \mathrm{mg} / \mathrm{dL}$ nos homens e $8,5 \mathrm{mg} / \mathrm{dL}$ nas mulheres, ambos valores considerados na faixa de normalidade. Conclusão: O gênero feminino possuía predomínio na realização da suplementação de cálcio e vitamina $D$, porém apresentava valores menores que o gênero masculino na última dosagem bioquímica.

\section{DESCRITORES}

Cirurgia Bariátrica. Obesidade. Suplementação. Nutrição.

\begin{abstract}
Objective: To evaluate the effect of calcium and vitamin D supplementation in individuals undergoing bariatric surgery. Methodology: This was a descriptive study, with 52 individuals, of both genders, with no age factor, being submitted to gastric bypass $Y$ of Roux type surgery, with a minimum postoperative time of 12 months. Results: It was found that $28.84 \%$ of individuals responded that they supplemented calcium and $57.69 \%$ supplemented vitamin D. In the comparison between genders, $7.69 \%$ of men and $35.89 \%$ of women reported supplemental calcium, $30.76 \%$ of men and $66.66 \%$ of women responded to supplement vitamin $\mathrm{D}$. The biochemical result of the last dose of vitamin D demonstrated men with $43.3 \mathrm{ng} / \mathrm{mL}$ and women with $32.6 \mathrm{ng} / \mathrm{mL}$, the determination of calcium had $9.1 \mathrm{mg} / \mathrm{dL}$ in men and $8.5 \mathrm{mg} / \mathrm{dL}$ in women, both values considered in the normal range. Conclusion: The female gender had a predominance in calcium and vitamin $D$ supplementation but presented lower values than the male gender in the last biochemistry dosage.
\end{abstract}

\section{DESCRIPTORS}

Bariatric Surgery. Obesity. Supplementation. Nutrition.

${ }_{1}^{1}$ Docente do Departamento de Ciências da Saúde, Curso de Fisioterapia, Universidade Paranaense, Toledo-PR.

2 Discentes do Curso de Fisioterapia da Universidade Paranaense, Toledo-PR. 
A obesidade é um problema global, sendo considerada uma epidemia mundial, que afeta aproximadamente 500 milhões de pessoas no mundo, estando fortemente associada a uma série de condições clínicas, como a dislipidemia, o diabetes mellitus tipo 2, a hipertensão arterial e as doenças cardiovasculares ${ }^{1,2}$.

Nos Estados Unidos foram registrados mais de 15 milhões de pessoas obesas graves, compondo o país de maior incidência dessa doença. No Brasil, calcula-se que existam 3,7 milhões de indivíduos acima do peso ideal. Acredita-se que, futuramente no mundo, 2,3 bilhões de pessoas estarão com parâmetros de sobrepeso e mais de 700 miIhões com diagnóstico de obesidade, fazendo desta disfunção uma das principais causas de mortes prematuras ${ }^{3,4}$.

Ocasionada devido ao armazenamento excessivo de gordura corporal em relação à massa magra é facilmente evidenciada na prática clínica por meio do cálculo do índice de massa corporal (IMC), obtido pela divisão do peso corporal, em quilogramas, pelo quadrado da altura, em metros ${ }^{5}$. Valores entre 30 e 34,9 $\mathrm{kg} / \mathrm{m}^{2}$ classificam a obesidade grau I, entre 35 e $39,2 \mathrm{~kg} / \mathrm{m}^{2}$ a obesidade grau II e acima de $40 \mathrm{~kg} / \mathrm{m}^{2}$ a obesidade grau III².

A indicação para o tratamento cirúrgico da obesidade deve basear-se em vários aspectos clínicos, avaliados por uma equipe multidisciplinar, envolvendo índice de massa corporal igual ou acima de $40 \mathrm{~kg} / \mathrm{m}^{2}$ ou superior a $35 \mathrm{~kg} / \mathrm{m}^{2}$, associado à comorbidades ${ }^{6,7}$.

Desse modo, a cirurgia bariátrica ou gastroplastia, como nominada, é a ferramenta mais eficaz no controle e tratamento da obesidade acentuada em indivíduos que já tentaram a perda de peso sem sucesso por meio de métodos conservadores, como as dietas hipocalóricas, a prática de atividade física regular e o uso de farmacoterapia. O procedimento vem crescendo de modo expressivo no mundo todo, registrando um aumento de $90 \%$ nos últimos cinco anos e $300 \%$ nos últimos dez anos. Apresenta benefícios que incluem resolução ou melhora acentuada das doenças crônicas, no entanto, é necessário salientar que o tratamento cirúrgico não se resume apenas ao ato operatório e, sim, em uma mudança comportamental dos hábitos alimentares e do estilo de vida ${ }^{3,8}$.

O preparo do indivíduo para a cirurgia exige uma adequada laboração da equipe multidisciplinar, tanto no pré quanto no pós-operatório, conduzindo a uma avaliação diagnóstica e tratamento adequado, individual, conjugal ou familiar, com orientações específicas sobre a cirurgia, visando discutir e adequar as expectativas do paciente às limitações do tratamento cirúrgico ${ }^{9}$.

O procedimento cirúrgico mais utilizado no Brasil para redução de massa corporal é tipo Fobi-Capella, gastroplastia Y de Roux, considerado o mais efetivo para o controle da obesidade mórbida ${ }^{8}$. É um procedimento misto, que promove saciedade precoce em decorrência da limitação gástrica e restrita absorção nutricional, pelo desvio do intestino delgado promovendo importante redução de peso ${ }^{10}$.

Alguns problemas nutricionais pós-operatórios, como a desnutrição proteica e a deficiência de vitaminas e minerais, ocorrem devido à redução do consumo alimentar e a menor absorção dos nutrientes. Estudos clínicos revelaram que, após a operação, 
ocorre uma perda de peso rápida, que pode se estender entre 18 e 24 meses, se instalando após este período disfunções nutricionais importantes ${ }^{6,11}$.

As principais deficiências nutricionais são de proteínas, ferro, vitamina B12, cálcio e vitamina $D$, sendo consideradas as deficiências desses micronutrientes as principais alterações que colocam em risco o sucesso do procedimento cirúrgico para o controle da obesidade $^{3,7}$.

A vitamina $D$, embora denominada uma vitamina lipossolúvel, se trata de um pré-hormônio por ser sintetizada através da pele, que juntamente com o paratormônio (PTH), atua como importante reguladora da homeostase do cálcio e do metabolismo ósseo ${ }^{1}$.

No processo de redução de peso pelo método cirúrgico está associada à restrição alimentar uma ingestão inadequada de cálcio, influenciando no eixo Cálcio-PTH com aumento na atividade osteoclástica ${ }^{3}$.

A deficiência de vitamina $D$ é caracterizada pela inadequada mineralização ou desmineralização do esqueleto, promovida de forma assintomática. Essa deficiência leva à diminuição dos níveis de cálcio ionizado, causando uma elevação na produção e secreção do paratormônio, o que provoca um aumento da reabsorção óssea, a fim de liberar cálcio para a corrente sanguínea e manter a homeostase do cálcio, condição conhecida como hiperparatireoidismo secundário ${ }^{12,13}$.

A diminuição severa de vitamina $D$ promove consequências graves à saúde óssea, envolvendo o risco de desenvolver a osteoporose, osteoartrose e fraturas ${ }^{13,14}$.

Complicações metabólicas e déficits nutricionais, como a baixa concentração de cálcio e da vitamina $D$, são comuns nos pacientes bariátricos. A adequada orientação, monitoramento e acompanhamento da equipe multidisciplinar são fundamentais na identificação e/ou na prevenção destas deficiências. Todos os profissionais devem estar atentos aos sinais e sintomas clínicos e ao monitoramento laboratorial rotineiro ${ }^{15}$.

Assim sendo, o objetivo deste estudo foi realizar uma abordagem da suplementação de cálcio e vitamina $D$ em indivíduos submetidos à cirurgia bariátrica.

\section{METODOLOGIA}

Foi realizado um estudo descritivo, transverasal, envolvendo indivíduos pósoperados, pela técnica de cirurgia bariátrica do tipo Fobi Capella com derivação em $Y$ de Roux, sem fator idade, de ambos os gêneros, submetidos ao procedimento há mais de 12 meses, participantes regulares das reuniões mensais oferecidas pelo grupo de apoio ao paciente bariátrico de uma clínica privada da Cidade de Toledo-PR, entre os meses de fevereiro a agosto de 2017.

Os critérios de inclusão envolveram indivíduos jovens e adultos, com histórico de acompanhamento clínico regular no pósoperatório e de exclusão, realização de outro tipo de gastroplastia, contra indicação de suplementação nutricional e ausência de exames bioquímicos atuais (últimos 6 meses) das concentrações de cálcio e de vitamina $D$.

Foi utilizado na coleta de dados um questionário semiestruturado que abordava identificação pessoal (nome, idade e gênero), estado civil (casado e solteiro), informações socioeconômicas (renda familiar mensal, com 
base no salário mínimo vigente da época), escolaridade (ensino fundamental, médio ou superior incompleto ou completo), peso e estatura pré-operatória e atual, tempo de pós-operatório e questões que investigavam a carência de cálcio e da vitamina $D$, prescrição clínica e realização de suplementação e modificações decorrentes do uso dos nutrientes, referidas por meio de exames bioquímicos, de cálcio pelo método de Arsenazo III e da vitamina $\mathrm{D}$ pelo método de imunoensaio quimioluminescente de micropartículas.

O questionário foi entregue em mãos pelo mesmo examinador, explicado, prontamente respondido e colhido a seguir, em horário antecedente a reunião mensal.

Após a coleta de dados, foi calculado o Índice de massa corporal (IMC), por meio das variáveis peso e estatura descritos no questionário. Os dados foram analisados utilizando-se o software Excel e Bioestat 5.0, por meio do cálculo da média, desvio padrão e porcentagem.

Todos os participantes assinaram o Termo de consentimento livre e esclarecido e o estudo foi aprovado pelo Comitê de Ética Envolvendo Seres Humanos da Universidade Paranaense, com Parecer consubstanciado $\mathrm{n}^{\circ}$. 1.177.620/2015.

\section{RESULTADOS}

Foram avaliados 52 indivíduos, sendo $75 \%$ do gênero feminino e $25 \%$ do gênero masculino, com média de idade de $38,5 \pm 11,55$ anos e média de pós-operatório de $31,25 \pm 11,35$ meses. Foi evidenciada uma média de peso antes da cirurgia de 109,80 $\pm 15,73$ $\mathrm{kg}$ e após de $74,69 \pm 16,03 \mathrm{~kg}$, resultando na média do índice de massa corporal (IMC) pré-operatório de $41,1 \pm 4,36 \mathrm{~kg} / \mathrm{m}^{2}$, reduzindo para $27,9 \pm 4,54 \mathrm{~kg} / \mathrm{m}^{2}$ pós-cirurgia. Na análise do perfil, a amostra foi composta por $61,5 \%$ casados, $36,5 \%$ solteiros e $1,9 \%$ que não se manifestaram. A pesquisa da renda familiar registrou $15,3 \%$ entre 1-2 salários-mínimos, $48,0 \%$ entre $3-4$ salários mínimos, $32,6 \%$ superior a 5 salários mínimos e 3,8\% não se manifestaram. O nível de escolaridade evidenciou $21,1 \%$ com ensino fundamental, $32,6 \%$ com ensino médio, 40,3\% com ensino superior e 5,7\% não se manifestaram (Tabela 1 ).

$\mathrm{Na}$ avaliação da realização da suplementação de cálcio, observou-se que 15 indivíduos (28,84\%) não suplementavam, 31 $(59,61 \%)$ faziam uso de suplementação e 6 $(11,53 \%)$ não se manifestaram (Figura 1).

$\mathrm{Na}$ comparação entre os gêneros, feminino e masculino, sobre a realização de suplementação de cálcio, evidenciou-se que $14(35,89 \%)$ mulheres e apenas $1(7,69 \%)$ homem relataram suplementar (Figura 2).

$\mathrm{Na}$ avaliação sobre a realização de suplementação de vitamina D obteve-se como resultado a afirmação de suplementação em $30(57,69 \%)$ avaliados (Figura 3$)$.

$\mathrm{Na}$ análise da suplementação de vitamina $D$ entre os gêneros, evidenciou-se que $4(30,76 \%)$ homens e $26(66,66 \%)$ mulheres referiram suplementar (Figura 4).

$\mathrm{Na}$ avaliação da última dosagem bioquímica de cálcio e da vitamina $\mathrm{D}$ em ambos os gêneros, constatou-se uma média de cálcio de $8,7 \pm 0,88 \mathrm{mg} / \mathrm{dL}$ e da vitamina $\mathrm{D}$ de $35,3 \pm 15,77 \mathrm{ng} / \mathrm{mL}$ (Figura 5).

Quanto à averiguação entre os gêneros da última dosagem bioquímica da vitamina $D$, constatou-se que os homens apresentaram 
Tabela 1. Perfil sociodemográfico dos indivíduos submetidos à cirurgia bariátrica em clínica privada de Toledo-PR

\begin{tabular}{lll}
\hline \multicolumn{1}{c}{ Variáveis } & $\mathrm{n}$ & $\%$ \\
\hline Gênero & 39 & 75 \\
Feminino & 13 & 25 \\
$\quad$ Masculino & 11 & 21,1 \\
Escolaridade & 17 & 32,6 \\
$\quad$ Ensino fundamental & 21 & 40,3 \\
Ensino médio & 03 & 5,7 \\
Ensino superior & & \\
Não se manifestaram & 08 & 15,3 \\
Renda Familiar & 25 & 48,0 \\
1-2 SM & 17 & 32,6 \\
3-4 SM & 02 & 3,8 \\
5 SM & & \\
Não se manifestaram & 32 & 61,5 \\
Estado Civil & 19 & 36,5 \\
Casado (a) & 01 & 1,9 \\
Solteiro (a) & Não se manifestaram & \\
\hline *SM - Salário mínimo vigente no período da coleta de dados de $\mathrm{R} \$ 937,00$
\end{tabular}

Figura 1. Suplementação de cálcio em indivíduos submetidos à cirurgia bariátrica em clínica privada de Toledo-PR

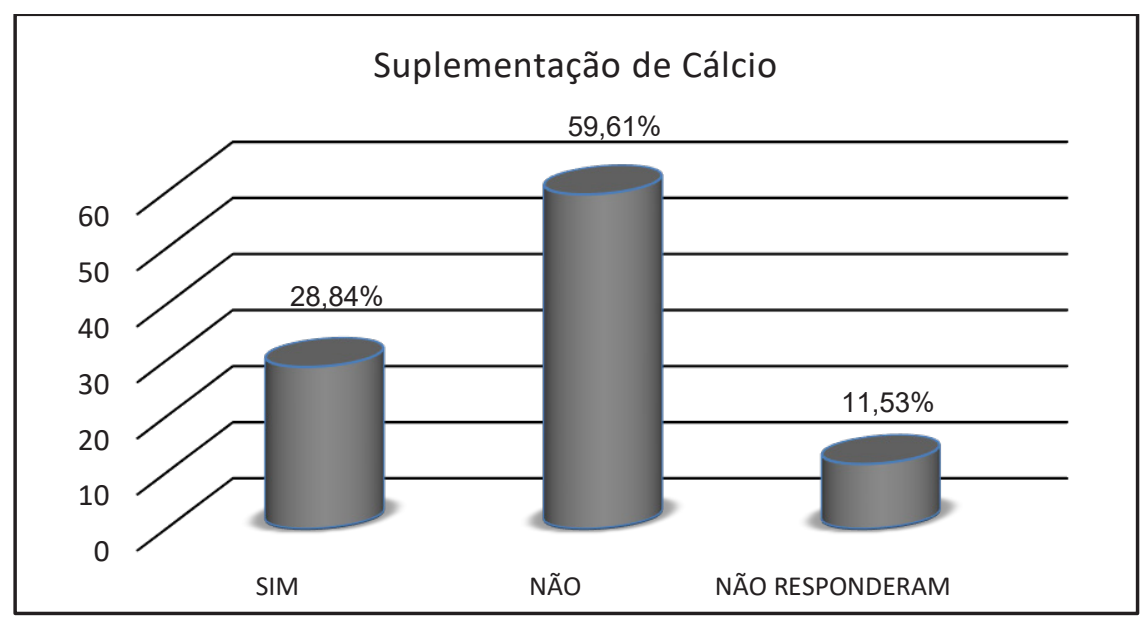


Figura 2. Suplementação de cálcio entre gêneros de indivíduos submetidos à cirurgia bariátrica em clínica privada de Toledo-PR

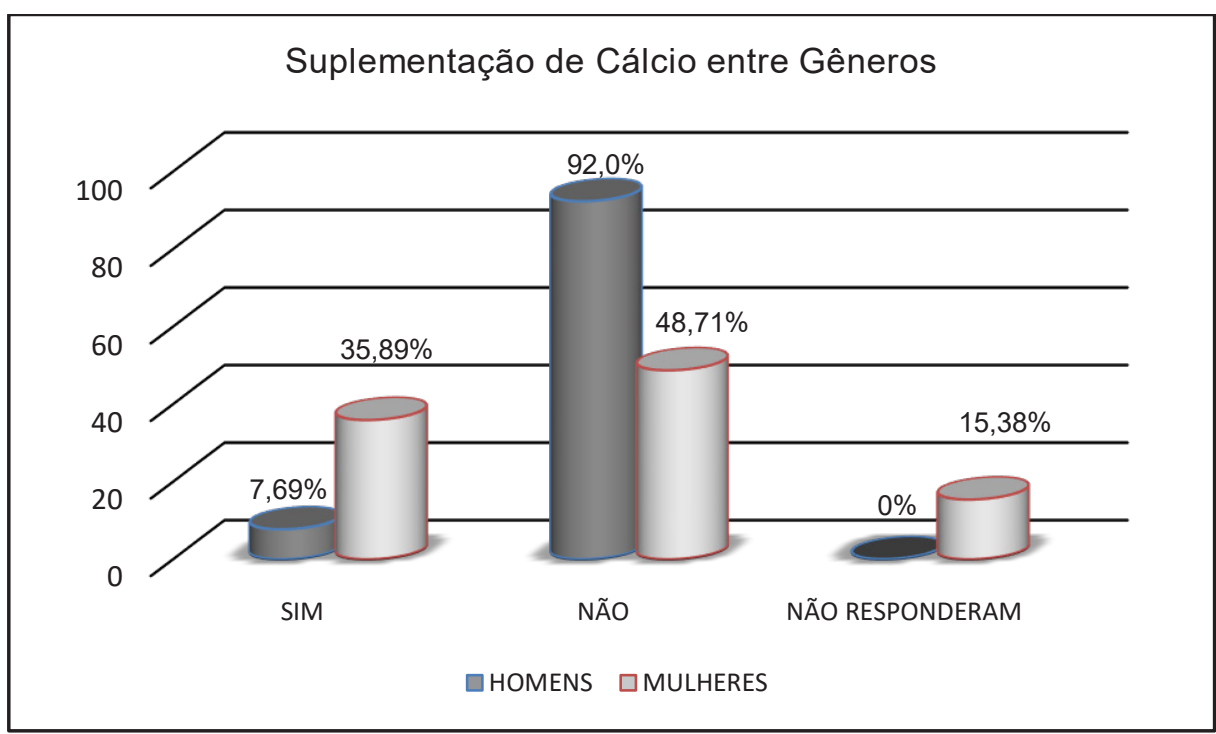

Figura 3. Suplementação de vitamina $\mathrm{D}$ em indivíduos submetidos à cirurgia bariátrica em clínica privada de Toledo-PR

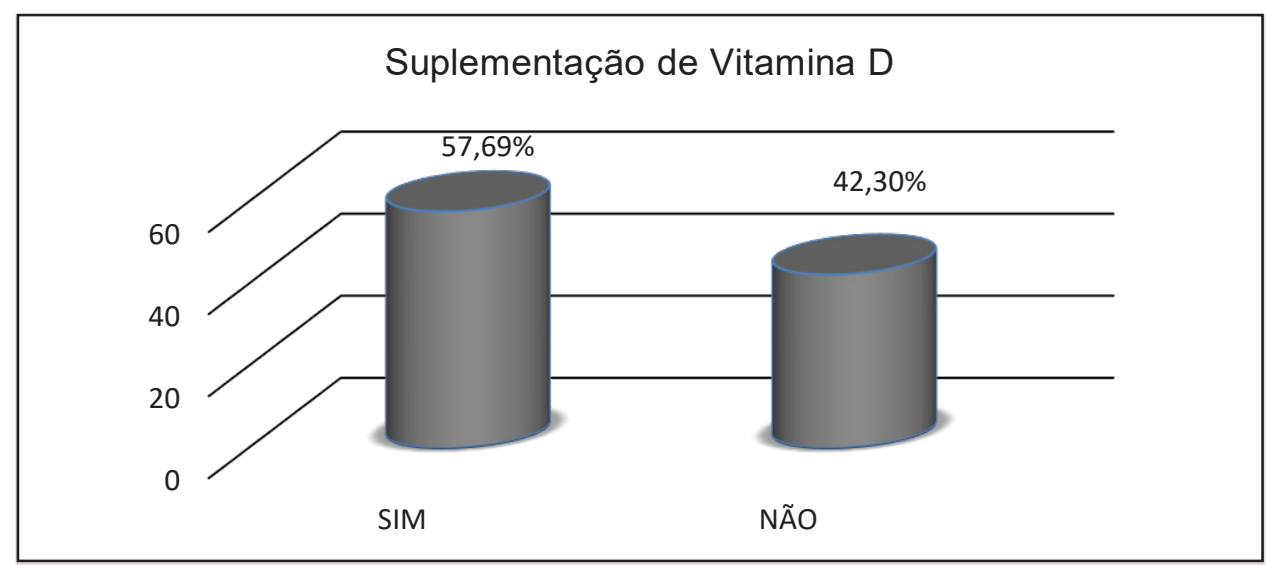

uma média de $43,3 \mathrm{ng} / \mathrm{mL}$ e as mulheres de 32,6ng/mL (Figura 6).

A análise da última dosagem bioquímica do cálcio entre os gêneros evidenciou valores de $9,1 \mathrm{mg} / \mathrm{dL}$ nos homens e $8,5 \mathrm{mg} / \mathrm{dL}$ nas mulheres (Figura 7).

\section{DISCUSSÃO}

Um estudo do tipo coorte prospectivo com 44 indivíduos, obesos mórbidos, operados com derivação gástrica em $Y$ de Roux e com anel de silicone, objetivando analisar as 
jura 4. Suplementação de vitamina $D$, entre gêneros, de indivíduos submetidos à cirurgia riátrica em clínica privada de Toledo-PR

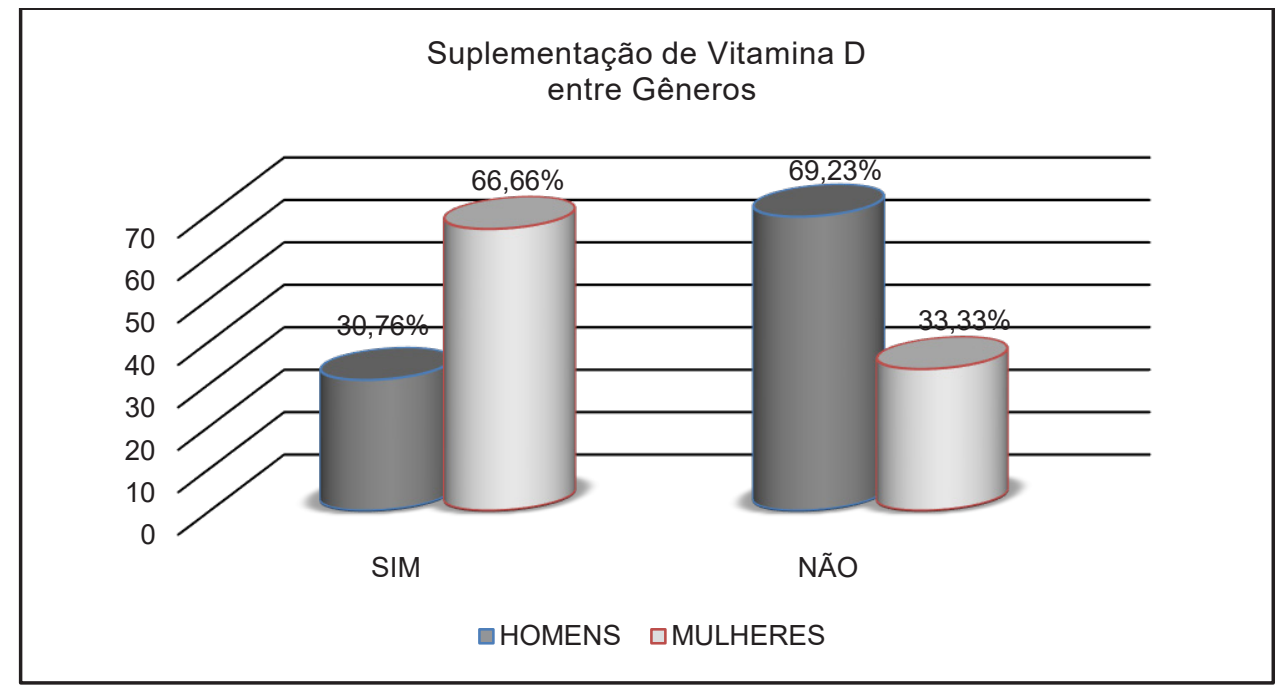

Figura 5. Valores da média da última dosagem bioquímica da vitamina $D$ e do cálcio de indivíduos submetidos à cirurgia bariátrica em clínica privada de Toledo-PR

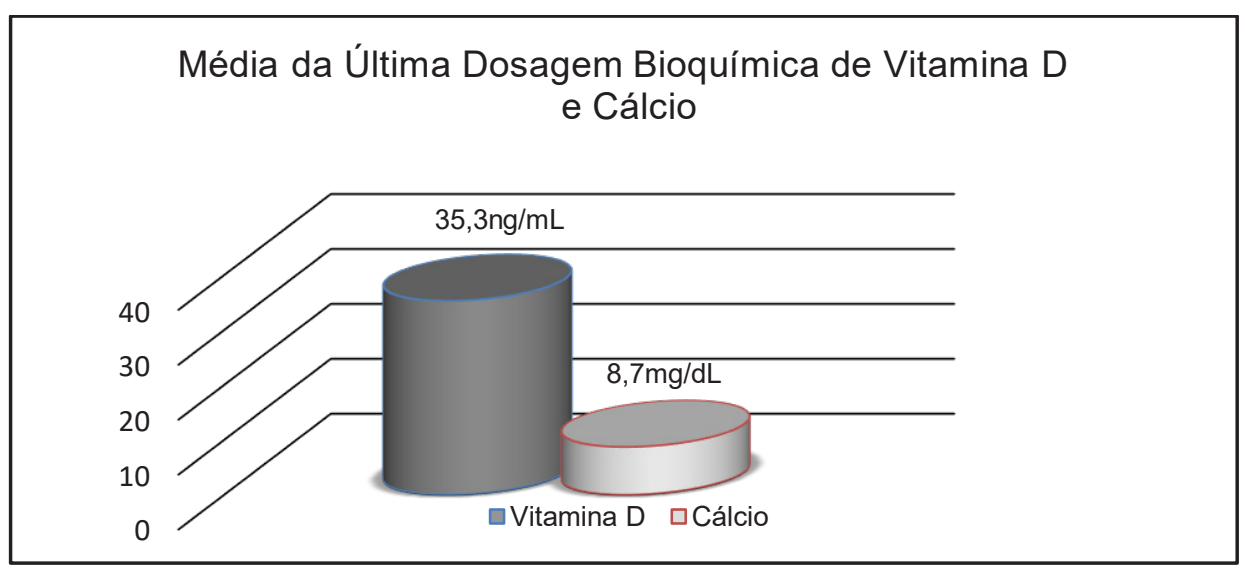

alterações sistêmicas decorrentes da redução de peso ao longo do tempo foi composto por $22,7 \%$ homens e $77,3 \%$ mulheres $^{16}$. Objetivando traçar um perfil clínico de 50 pacientes candidatos à cirurgia bariátrica de um hospital de Manaus-AM evidenciou-se $78 \%$ do gêne- ro feminino e $22 \%$ do gênero masculino ${ }^{2}$. O presente estudo também obteve uma predominância do público feminino (75\%) no estudo sobre cirurgia bariátrica.

A média da idade dos pesquisados neste estudo foi de $38,5 \pm 11,55$ anos, sendo 
Figura 6. Média entre os gêneros da última dosagem bioquímica da vitamina $D$ em indivíduos submetidos à cirurgia bariátrica em clínica privada de Toledo-PR

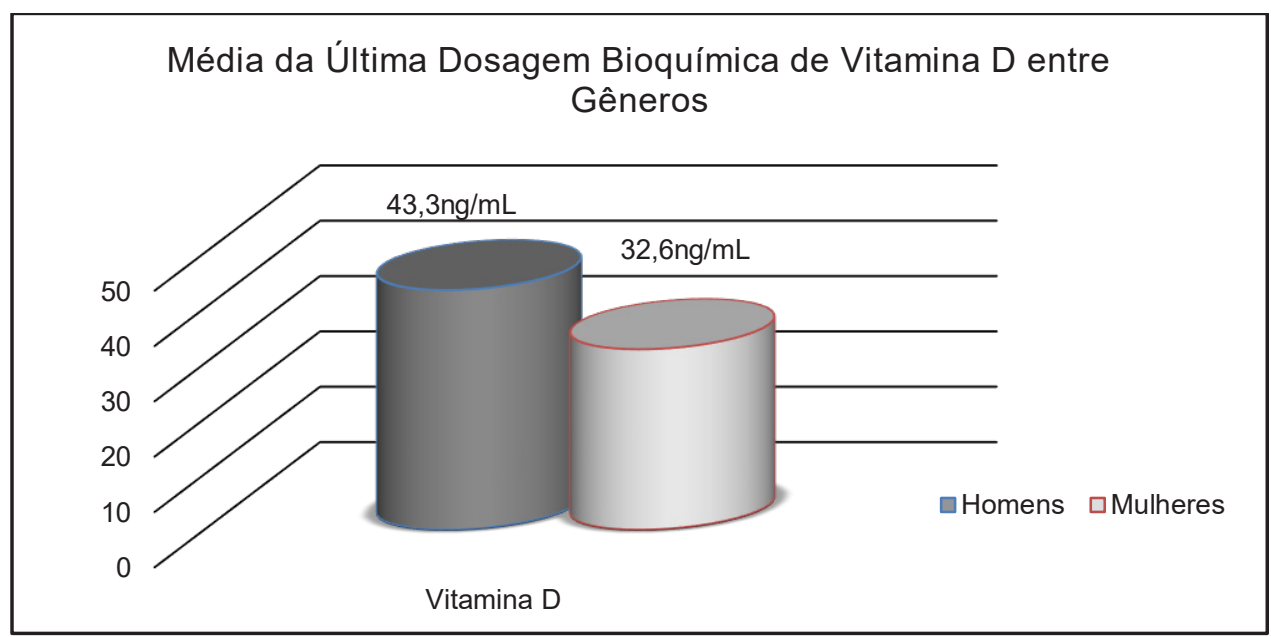

Figura 7. Média entre os gêneros da última dosagem bioquímica do cálcio em indivíduos submetidos à cirurgia bariátrica em clínica privada de Toledo-PR

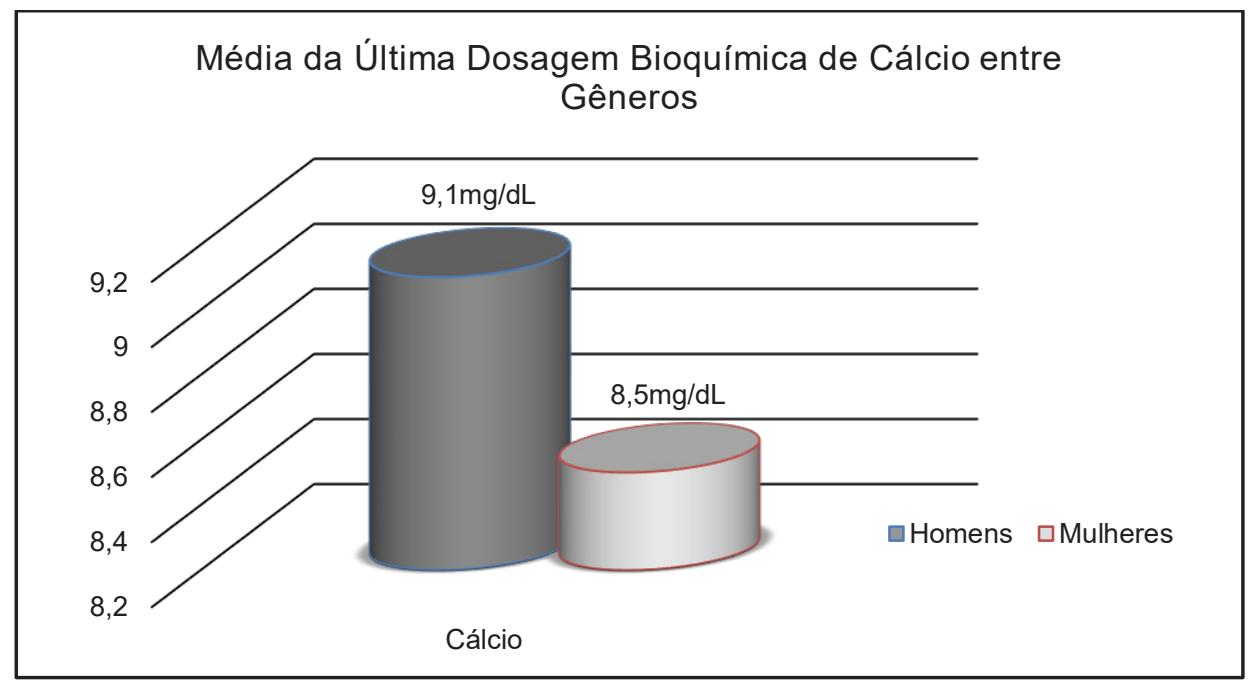

a idade mínima de 19 e máxima de 58 anos. Aproximando-se do resultado de estudo realizado recentemente, sendo a idade mínima de 18 e máxima de 59 anos, o que salienta maiores riscos de morbimortalidade na obesidade em indivíduos adultos em idade produtiva ${ }^{2}$.
Avaliando a adequação de micronutrientes da dieta após gastroplastia em 36 mulheres, observou-se na amostra um nível de escolaridade de $22,2 \%$ com ensino fundamental incompleto, $11,1 \%$ fundamental completo, $8,3 \%$ médio incompleto, $41,7 \%$ médio 
completo, 5,6\% superior incompleto e $11,1 \%$ superior completo ${ }^{17}$. Dados evidenciados no presente estudo denotaram que a maioria $(40,3 \%)$ possuía ensino superior completo/ incompleto seguido de $32,6 \%$ com ensino médio completo/incompleto $32,6 \%$.

Objetivando analisar o perfil nutricional e a perda de peso de indivíduos bariátricos, constatou-se que a maioria era composta por mulheres $(86,1 \%)$, sendo $50 \%$ casadas $^{10}$. Achados semelhantes ao deste estudo em que a maioria $(50 \%)$ também assinalaram como estado civil a opção casada.

Ao investigar a qualidade de vida de 30 indivíduos submetidos à cirurgia bariátrica apurou-se uma renda familiar de 16,66\% entre 1-2 salários mínimos, 43,33\% 3-4 salários mínimos e $40 \%$ renda superior a 5 salários mínimos ${ }^{8}$, corroborando com o presente estudo, em que a maioria $(48,07 \%)$ também estava na faixa de 3-4 salários mínimos.

Em paralelo aos índices epidêmicos de sobrepeso e obesidade, observou-se a popularização da cirurgia bariátrica no estudo que descreveu indivíduos com valores de IMC entre 50 e $51 \mathrm{~kg} / \mathrm{m}^{2}$ no pré-operatório ${ }^{2}$. Outro estudo, de 2016, com indivíduos submetidos à gastroplastia do tipo $Y$ de Roux, referiu em $47,5 \%$ dos indivíduos valores mais expressivos $^{10}$ que a média de $41,1 \mathrm{~kg} / \mathrm{m}^{2}$ descrita neste estudo, ambos valores indicativos de tratamento da obesidade por meio de procedimento cirúrgico.

A técnica cirúrgica de redução de peso mais utilizada no Brasil é a do tipo Bypass gástrico em Y de Roux. É definida por um procedimento que altera o trato gastrointestinal, levando à redução da ingestão calórica e à redução da absorção dos alimentos. É uma técnica restritiva que reduz a capacidade gástrica do estômago, levando à redução calórica por meio da saciedade precoce, entretanto, possui como eventuais complicações carências nutricionais, sobretudo evidenciadas após a estabilização do peso ${ }^{15}$. Neste estudo, abordando a cirurgia descrita, foi possível observar a necessidade de suplementação nutricional, sobretudo em mulheres que demonstraram maior vulnerabilidade à carência de vitamina D e do cálcio.

Quanto aos hábitos alimentares de 83 pacientes submetidos à cirurgia bariátrica denotou-se uma ingesta de cálcio abaixo dos valores recomendados diariamente, enfatizando que a ingesta diária de indivíduos adultos deve ser proporcional a $1.000 \mathrm{mg}$, fazendo-se necessário o uso da suplementação ${ }^{18}$. Dados evidenciados nesta pesquisa diante da necessidade de suplementação de cálcio em um percentual expressivo do público estudado.

Um estudo destaca que a suplementação de cálcio na forma de citrato de cálcio é preferível, pois a ausência de produção ácida no estômago prejudica a absorção na forma de carbonato, sendo considerado normal para indivíduos adultos valores entre 8,5 a 10,5mg/ $\mathrm{dL}^{15}$. Neste estudo, por meio da avaliação da última dosagem bioquímica de cálcio, apurou-se que as mulheres apresentaram uma média de $8,5 \mathrm{mg} / \mathrm{dL}$ e os homens $9,1 \mathrm{mg} / \mathrm{dL}$, notificando que a suplementação possui efeito positivo na normalização do nutriente.

A suplementação da vitamina $D$ e do cálcio pode refletir na manutenção da massa óssea em pacientes pós-cirurgia bariátrica, sendo considerados fatores importantes a idade e o gênero analisado. Mulheres, principalmente, após a menopausa, são mais 
susceptíveis às carências nutricionais do que os homens ou mulheres mais jovens ${ }^{14,19}$, o que confirma os achados deste estudo, quanto à necessidade e a realização de suplementação ser maior entre as mulheres.

A vitamina $D$ está envolvida diretamente na absorção e no metabolismo do cálcio, a deficiência dessa vitamina pode ocorrer após a cirurgia bariátrica, visto que a principal região de absorção desse micronutriente, que é no duodeno e no jejuno proximal, fica excluída do transito alimentar, devido ao desvio intestinal realizado. São considerados valores normais entre 30 e $44 \mathrm{ng} / \mathrm{mL}$ comprovados em exames bioquímicos ${ }^{12,17}$. Valores evidenciados no presente estudo, porém com maiores quantidades constatadas no gênero masculino.

A absorção deficitária de minerais e de vitaminas lipossolúveis tem sido relatada em indivíduos de ambos os gêneros após o procedimento de cirurgia bariátrica, sobretudo, o cálcio e a vitamina $D$, essenciais para a integridade da saúde óssea. Quanto maior a perda de peso mais inegável é a deficiência de alguns nutrientes. Diante da diminuição de vitamina $D$ no organismo a absorção do cálcio fica comprometida, promovendo casos importantes de osteoporose, evidenciados em ossos que se estendem dos membros inferiores à arcada dentária ${ }^{7,20,21}$.

Estima-se que $80 \%$ a $90 \%$ da vitamina D existente no corpo, sejam sintetizadas pela ação da luz ultravioleta em 7-dehidrocolesterol e, os $10 \%$ a $20 \%$ restantes, sejam adquiridos pela ingestão de alimentos fontes desta vitamina, como gema de ovo, laticínios e peixes gordurosos ${ }^{3}$. Portanto, torna-se necessário o acompanhamento da equipe multidisciplinar, quanto à busca por hábitos mais saudáveis, na garantia da perda de peso de forma segura.

Um estudo envolvendo 15 pacientes submetidos à gastroplastia comprovou uma diminuição considerável, em torno de $25 \%$, na absorção da suplementação de vitamina $D$ na forma de colecalciferol, complicando ainda mais qual seria o melhor tipo de suplemento e qual seria a dose diária recomendada. Entretanto, estimou-se que um paciente pós-cirurgia bariátrica necessite de doses quatro a oito vezes maiores do que um indivíduo normal. Não existe, ao certo, um período correto de início ou agravamentos das deficiências, assim, não existe o momento exato para iniciar a suplementação, bem como, se torna complexo definir a dose ideal para cada indivíduo, pois cada metabolismo reage de forma diferenciada ${ }^{22}$.

O monitoramento das concentrações de cálcio e da vitamina $D$ é fácil de ser realizada, como também, a suplementação destes nutrientes, sendo fatores decisivos para atenuar a perda de massa óssea após a cirurgia bariátrica. A doença óssea metabólica é uma complicação em longo prazo, por isso torna-se imprescindível reforçar que o acompanhamento deste paciente deve ser permanente ${ }^{11,15}$.

\section{CONCLUSÃO}

O presente estudo evidenciou que a busca do tratamento da obesidade por meio da cirurgia bariátrica é maior em indivíduos adultos, mulheres, sendo importante a realização de pesquisas que investiguem não somente a solução da doença e suas comorbidades, bem como, as alterações metabólicas provocadas no pós-operatório. 
$\mathrm{Na}$ análise sobre a suplementação da vitamina D constatou-se que $57,69 \%$ faziam uso de suplementação, sendo predominante o gênero feminino. Na avaliação da suplementação do cálcio evidenciou-se que $28,84 \%$ faziam uso, com predomínio também das mulheres.

$\mathrm{Na}$ análise da última dosagem bioquímica do cálcio e da vitamina $D$ foi possível averiguar que o gênero masculino demonstrou dosagem superior que o feminino, embora,

\section{REFERÊNCIAS}

1. Schmidt A. Relação entre a deficiência de vitamina $D$ e obesidade: uma revisão atual. Rev. Bras. Obes. Nutr. Emagr. 2015; 9(53):207-212.

2. Silva CDA, Figueira MA, Maciel MCSPG, Gonçalves RL, Sanchez FF. Perfil clínico de pacientes candidatos à cirurgia bariátrica. Rev. Bras. Obes. Nutr. Emagr. 2017;11(64):211-216.

3. Mustafa S. A cirurgia bariátrica e a perda óssea. Int J Nutrol. 2014;7(1):22-30.

4. Rey-López JP, Rezende LF, Sá TH, Stamatakis E. Is the metabolically healthy obesity phenotype an irrelevant artifact for public health? Am J Epidemiol. 2015;182(9):737741.

5. Freitas AR, Vicente MA. Avaliação das alterações nutricionais, clinicas e bioquímicas em pacientes submetidos a cirurgia bariátrica nos períodos pré e pós-cirúrgico. Rev. Bras. Obes. Nutr. Emagr. 2011;5(28):333-343.

6. Silva PRB, Souza MR, Silva EM, Silva SA. Estado nutricional e qualidade de vida em pacientes submetidos à cirurgia bariátrica. Arq. Bras. Cir.Dig. 2014;27(1):35-38.

7. Segura DCA, Wozniak SD, Andrade FL, Marreto TM, Ponte ED. Deficiências nutricionais e suplementação em indivíduos submetidosà gastroplastia redutora do tipo $\mathrm{Y}$ de Roux. Rev. Bras.Obes. Nutr. Emagr. 2017;11(65):338347 .

8. Segura DCA, Nascimento FC, Beuren TR, Wozniak SD. Análise da qualidade de vida de pacientes submetidos à cirurgia bariátrica e hábitos de atividade física pós-cirurgia. Braz J SurgClin Res. 2015; 9(1):12-19.

9. Moraes JM, Caregnato RCA, Schneider DS. Qualidade de vida antes e após a cirurgia bariátrica. Acta Paul. Enfer. 2014; 27(2):157-164 em ambos os gêneros os parâmetros foram de normalidade, norteando a importância da suplementação de forma profilática.

Concluindo, o gênero feminino possuía predomínio na realização da suplementação do cálcio e da vitamina $D$, porém, apresentava menores valores na última dosagem bioquímica. Consequentemente, mesmo os homens suplementando menos, exibiram melhores resultados bioquímicos comparados ao gênero oposto.

10. Oliveira CCA, Pinto SL. Perfil nutricional e perda de peso de pacientes submetidos à cirurgia de bypass gástrico em Y de Roux. Rev. Bras.Rev. Bras. Nutr. Clin. 2016; 31(1):18-22.

11. SantosHN,Lima JMS, Souza MFC. Estudo comparativo da evolução nutricional de pacientes candidatos à cirurgia bariátrica assistidos pelo Sistema Único de Saúde e pela Rede Suplementar de Saúde. Ciênc Saúde Col. 2014;19(5):1359-1365.

12. Rosen CJ, Adams JS, Bikle DD, Black DM, Demay MB Manson JE, Murad MH, Kovacs CS. The nonskeletal effects of vitamin D: An endocrine society scientific statement. Endocr Rev. 2012; 33(3):456-492.

13. Peters BSE, Martini LA. Funções plenamente reconhecidas de nutrientes-vitamina D. ILSI Brasil. 2014:2(2):1-19.

14. Scibora LM, Ikramuddin S, Buchwald H, Petit MA. Examining the link between bariatric surgery. Bone loss and osteoporosis: A review of bone density studies.ObesSurg. 2012; 22:654-667

15. Ramos CP, Mello ED. Manejo nutrológico no pós-operatório de cirurgia bariátrica. Int J Nutrol. 2015;8(2):39-49.

16. Jóia Neto L, Lopes Junior AG, Jacob CE. Alterações metabólicas e digestivas no pós-operatório de cirurgia bariátrica. Arq Bras Cir Dig. 2010; 23(4):266-269.

17. Leiro LS, Araújo MSM. Adequação de micronutrientes da dieta de mulheres após um ano de by-pass gástrico. Arq Bras Cir Dig. 2014; 27(1):21-25.

18. Costa TMRL, Paganoto M, Radominski RB, Borba VZC Impacto da deficiência nutricional na massa óssea após cirurgia bariátrica. Arq Bras Cir Dig. 2016; 29(1):38-42. 
19. Cabral JAV, Souza GP, Nascimento JA, Simoneti LF, Marchese C, Peres SHCS. Impacto da deficiência da vitamina $\mathrm{D}$ e cálcio em ossos de pacientes submetidos á cirurgia bariátrica: uma revisão sistemática. Arq Bras Cir Dig. 2016; 29(1):120-123.

20. Fleischer J, Stein EM, Bessler M, DellaBadia M, Restuccia $\mathrm{N}$, Olivero-Rivera $\mathrm{L}$, et al. The decline in hip bone density after gastric bypass surgery is associated with extent of weight loss. J Clin Endocrinol Metab. 2008;93(10):37353740 .

21. Moura-Grec PG, Assis VH, Cannabrava VP, Vieira VM, Siqueira TLD, Anaguizawa WH, Sales-Peres SHC. Consequências sistêmicas da cirurgia bariátrica e suas Repercussões na saúde bucal. Arq Bras Cir Dig. 2012; 25(3):173-177.
22. Aarts E, Van Groningen L, Horst R, Telting D, Van Sorge A, Janssen I, de Boer H. Vitamin D absorption: consequences of gastric bypass surgery. Eur $\mathrm{J}$ Endocrinol. 2011;164:827-832.

\section{CORRESPONDÊNCIA}

Rua General Rondon, 2218, Jd. La Salle

Cep.85902-090Toledo-PR.

E-mail: dora@prof.unipar.br 